\title{
The Double Prior Selection for the Parameter of Exponential Life Time Model under Type II Censoring
}

Ronak M. Patel

Som-Lalit College of Commerce, Ahmedabad, ronak2307@yahoo.in

Achyut C. Patel

Smt.M. T. Dhamsania Community College, Rajkot, acp2809@gmail.com

Follow this and additional works at: http://digitalcommons.wayne.edu/jmasm

Part of the Applied Statistics Commons, Social and Behavioral Sciences Commons, and the Statistical Theory Commons

\section{Recommended Citation}

Patel, R. M. \& Patel, A. C. (2017). The double prior selection for the parameter of exponential life time model under Type II censoring. Journal of Modern Applied Statistical Methods, 16(1), 406-427. doi: 10.22237/jmasm/1493598180

This Regular Article is brought to you for free and open access by the Open Access Journals at DigitalCommons@WayneState. It has been accepted for inclusion in Journal of Modern Applied Statistical Methods by an authorized editor of DigitalCommons@WayneState. 


\title{
The Double Prior Selection for the Parameter of Exponential Life Time Model under Type II Censoring
}

\author{
Ronak M. Patel \\ Som-Lalit College of Commerce \\ Ahmedabad, India
}

\author{
Achyut C. Patel \\ Smt. M. T. Dhamsania Community College \\ Rajkot, India
}

\begin{abstract}
A comparison of double informative priors assumed for the parameter of exponential life time model is considered. Three different sets of double priors are included, and the results are compared with a forth single prior. The data is Type II censored and Bayes estimators for the parameter and reliability are carried out under a squared error loss function in the cases of the four different sets of prior distributions. The predictive distribution was derived for future failure time and also for the remaining ordered failure times after the first $r$ failure times have been observed. Corresponding Bayes credible equal tail intervals are also derived. Simulations and real data are employed to exemplify the method.
\end{abstract}

Keywords: Gamma prior, chi-square prior, predictive intervals, squared error loss function

\section{Introduction}

In life testing experiments, the experimenter may not be always in a position to observe the life times of all items tested because of time limitations or restrictions on the number of failures during the test due to very high-cost items. When the cost of the experiment is directly proportional to the number of failures, the failure-censored experiment is more preferable than the time-censored experiment. The failure-censored experiment is also known as Type II censoring. In this censoring scheme, the test is terminated as soon as the pre-determined number of failures $(r)$ is observed out of $n$ units tested.

The exponential distribution has an important position in life time models. It is the first lifetime model for which statistical methods were extensively developed. Many authors contributed to the methodology of this distribution.

Ronak M. Patel is a Lecturer in the Department of Statistics. Email him at: ronak2307@yahoo.in. 
Important works include Epstein (1954), Epstein (1960a, 1960b), Epstein and Sobel (1953), Epstein and Sobel (1954), Bartholomew (1957), Mann, Schafer, and Singpurvala (1974), Lawless (1971), Balakrishnan and Cohen (1991), and others. A number of authors considered prediction problems for the exponential distribution. For example, Hahn (1975), Lawless (1971), and Likeš (1974). In life testing experiments, prediction using the Bayesian method is considered by Box and Tiao (1973), Dunsmore (1974), Evans and Nigm (1980), and Howlader and Hossain (1995). Saleem and Aslam (2008), Tahir and Zawar (2008), and Haq and Dey (2011) considered comparison and selection of a suitable prior using Bayesian methodology.

They considered a set of single prior distributions for comparison. However, there may have been different prior information about the unknown parameter of the lifetime model; to include two different kind of information in the Bayesian analysis, two different priors have been selected for a single unknown parameter of the life time model. Haq and Aslam (2009) considered double prior selection for the parameter of a Poisson distribution based on posterior variance, posterior predictive variance, and the posterior predictive probabilities. Radha and Vekatesan (2013) considered the problem of selection of double priors for the parameter of a Maxwell distribution. They did not derive any Bayes estimator, but just showed that the double priors and their posterior distribution belong to the same family.

The purpose of the current study, therefore, is to contribute something in this direction of double prior distribution for the parameter of the exponential life time model. The following three different types of joint priors and one type of single prior are used for the unknown parameter $\theta$ of the exponential distribution:

(i) Exponential-Gamma distribution

(ii) Gamma-Chi-square distribution

(iii)Chi-square-Exponential distribution

(iv) Gamma distribution

Bayes estimators of parameter $\theta$ and reliability at time $t$ are obtained based on a Type II censored sample (with fixed $r$ observed failures) under squared error loss function based on the above prior distributions. Also, Bayes predictive estimation and Bayes predictive equal credible intervals are carried out. Prediction of the remaining $(n-r)$ failure times is done and their Bayes credible prediction intervals are also derived. A real-life example is considered to exemplify the 
theoretical results obtained in the paper, as is a simulation study, and comparison is made between the results obtained based on the different priors considered.

\section{The Posterior Distribution of $\theta$ Under Different Prior Distributions}

Let $n$ items be put on a life test such that the test is terminated as soon as the $r^{\text {th }}$ failure is observed, and the corresponding failure times are $X_{(1)}, X_{(2)}, \ldots, X_{(n)}$. During the test, failure items are not replaced, but the test is carried out with the remaining items on the test. Such a censoring scheme is known as Type II censoring without replacement. We assume the life time model is exponential with mean life $1 / \theta, \theta>0$. The probability density function (pdf) and the cumulative distribution function (cdf) of this life time model are, respectively, given by

$$
\begin{gathered}
\mathrm{f}(x, \theta)=\theta \mathrm{e}^{-\theta x}, \quad x>0, \theta>0 \\
\mathrm{~F}(x, \theta)=1-\mathrm{e}^{-\theta x}
\end{gathered}
$$

Its reliability function at time $t$ is given by

$$
\mathrm{R}_{t}(\theta)=\mathrm{e}^{-\theta t}, \quad t>0
$$

The likelihood function under such a censoring scheme is given by

$$
\mathrm{L}(x, \theta)=C \prod_{i=1}^{r} \mathrm{f}\left(x_{(i)}, \theta\right)\left[1-\mathrm{F}\left(x_{(r)}, \theta\right)\right]^{n-r}
$$

Using (1) and (2),

$$
\mathrm{L}(x, \theta)=C \theta^{r} \mathrm{e}^{-\theta\left[\sum_{i=1}^{r} x_{(i)}+(n-r) x_{(r)}\right]}
$$

\section{Exponential and Gamma Distributions as Double Priors}

Consider the first prior distribution of $\theta$ to be exponential with hyper parameter $c_{1}$, having pdf 


$$
\mathrm{p}_{11}(\theta)=c_{1} \mathrm{e}^{-c_{1} \theta}, \quad \theta>0, c_{1}>0
$$

The second prior distribution is a gamma distribution with hyper parameters $a_{1}$ and $b_{1}$, having pdf

$$
\mathrm{p}_{12}(\theta)=\frac{\mathrm{e}^{-b_{1} \theta} \theta^{a_{1}-1} b_{1}^{a_{1}}}{\Gamma\left(a_{1}\right)}, \quad \theta>0, b_{1}>0, a_{1}>0
$$

The double prior for $\theta$ can be defined by combining these two priors as follows:

$$
\mathrm{p}_{1}(\theta) \propto \mathrm{p}_{11}(\theta) \mathrm{p}_{12}(\theta)=k_{1} \mathrm{e}^{-\left(b_{1}+c_{1}\right) \theta} \theta^{a_{1}-1}
$$

where

$$
k_{1}=\frac{\left(c_{1}+b_{1}\right)^{a_{1}}}{\Gamma\left(a_{1}\right)}
$$

Hence, the posterior distribution of $\theta$ for the given data $x$ is obtained, using (4) and (7), as

$$
\pi_{1}(\theta \mid x)=\frac{\left(y+c_{1}+b_{1}\right)^{a_{1}+r}}{\Gamma\left(a_{1}+r\right)} \mathrm{e}^{-\left(y+b_{1}+c_{1}\right)^{\theta} \theta^{a_{1}+r-1}}, \quad \theta>0
$$

where

$$
y=\sum_{i=1}^{r} x_{(i)}+(n-r) x_{(r)}
$$

which is the gamma distribution with parameters $\alpha_{1}=r+a_{1}$ and $\beta_{1}=y+c_{1}+b_{1}$. That is, $\pi_{1}(\theta \mid x)$ has gamma $\mathrm{G}\left(\alpha_{1}, \beta_{1}\right)$ distribution.

\section{Gamma and Chi-Square Distributions as Double Priors}

Assume the first prior distribution for $\theta$ is a gamma distribution: 


\section{DOUBLE PRIOR SELECTION FOR EXPONENTIAL DISTRIBUTION}

$$
\mathrm{p}_{21}(\theta)=\frac{\mathrm{e}^{-b_{2} \theta} \theta^{a_{2}-1} b_{2}^{a_{2}}}{\Gamma\left(a_{2}\right)}, \quad \theta>0, b_{2}>0, a_{2}>0
$$

The second prior for $\theta$ is a chi-square distribution having pdf

$$
\mathrm{p}_{22}(\theta)=\frac{\mathrm{e}^{\frac{-\theta}{2}} \theta^{\left(\frac{c_{2}}{2}\right)-1}}{2^{\frac{c_{2}}{2}} \Gamma\left(\frac{c_{2}}{2}\right)}, \quad \theta>0, c_{2}>0
$$

By combining (9) and (10), obtain the double prior distribution for $\theta$ as

$$
\mathrm{p}_{2}(\theta) \propto \mathrm{p}_{21}(\theta) \mathrm{p}_{22}(\theta)=k_{2} \mathrm{e}^{-\left(b_{2}+\frac{1}{2}\right) \theta} \theta^{\left(a_{2}+\frac{c_{2}}{2}-1\right)-1}
$$

where

$$
k_{2}=\frac{\left(b_{2}+\frac{1}{2}\right)^{a_{2}+\frac{c_{2}}{2}-1}}{\Gamma\left(a_{2}+\frac{c_{2}}{2}-1\right)}
$$

Hence, the posterior distribution of $\theta$ based on this double prior distribution of $\theta$ for given data $x$ can be obtained, using (4) and (11), as

$$
\pi_{2}(\theta \mid x)=\frac{\left(y+b_{2}+\frac{1}{2}\right)^{a_{2}+\frac{c_{2}}{2}+r-1}}{\Gamma\left(a_{2}+\frac{c_{2}}{2}+r-1\right)} \mathrm{e}^{-\left(y+b_{2}+\frac{1}{2}\right) \theta} \theta^{\left(a_{2}+\frac{c_{2}}{2}+r-1\right)-1}, \quad \theta>0
$$

which is the gamma distribution $\mathrm{G}\left(\alpha_{2}, \beta_{2}\right)$, where

$$
\alpha_{2}=a_{2}+\frac{c_{2}}{2}+r-1, \quad \beta_{2}=y+b_{2}+\frac{1}{2}
$$




\section{Chi-Square and Exponential Distributions as Double Priors}

In a similar manner, assume both the prior distributions have pdfs given by

$$
\mathrm{p}_{31}(\theta)=\frac{\mathrm{e}^{-\frac{\theta}{2}} \theta^{\frac{c_{3}}{2}-1}}{2^{\frac{c_{3}}{2}} \Gamma\left(\frac{c_{3}}{2}\right)}, \quad \theta>0, c_{3}>0
$$

and

$$
\mathrm{p}_{32}(\theta)=b_{3} \mathrm{e}^{-b_{3} \theta}, \quad \theta>0, b_{3}>0
$$

Hence, the double prior distribution $\theta$ becomes

$$
\mathrm{p}_{3}(\theta)=k_{3} \mathrm{e}^{-\left(b_{3}+\frac{1}{2}\right) \theta} \theta^{\frac{c_{3}}{2}-1}
$$

where

$$
k_{3}=\frac{\left(b_{3}+\frac{1}{2}\right)^{\frac{c_{3}}{2}}}{\Gamma\left(\frac{c_{3}}{2}\right)}
$$

and the posterior distribution of $\theta$ given the data $x$, based on this double prior distribution, comes out to be

$$
\pi_{3}(\theta \mid x)=\frac{\left(y+b_{3}+\frac{1}{2}\right)^{\frac{c_{3}}{2}+r}}{\Gamma\left(\frac{c_{3}}{2}+r\right)} \mathrm{e}^{-\left(y+b_{3}+\frac{1}{2}\right) \theta} \theta^{\frac{c_{3}}{2}+r-1}, \quad \theta>0
$$

which is the gamma distribution $\mathrm{G}\left(\alpha_{3}, \beta_{3}\right)$, where

$$
\alpha_{3}=\frac{c_{3}}{2}+r, \quad \beta_{3}=y+b_{3}+\frac{1}{2}
$$




\section{Only Single Gamma $G\left(a_{4}, b_{4}\right)$ Prior Distribution for $\theta$}

Here we consider only a single gamma prior distribution for $\theta$, given by

$$
\mathrm{p}_{4}(\theta)=\frac{\mathrm{e}^{-b_{4} \theta} \theta^{a_{4}-1} b_{4}^{a_{4}}}{\Gamma\left(a_{4}\right)}, \quad \theta>0, b_{4}>0, a_{4}>0
$$

The corresponding posterior distribution for $\theta$ becomes

$$
\pi_{4}(\theta \mid x)=\frac{\left(y+b_{4}\right)^{a_{4}+r}}{\Gamma\left(a_{4}+r\right)} \mathrm{e}^{-\left(y+b_{4}\right) \theta} \theta^{a_{4}+r-1}, \quad \theta>0
$$

which is also a gamma distribution $\mathrm{G}\left(\alpha_{4}, \beta_{4}\right)$ with parameters $\alpha_{4}=r+a_{4}$ and $\beta_{4}=y+b_{4}$. Thus, in all the cases of the different types of double prior distributions and in the case of a single prior distribution, the posterior distribution of $\theta$ given the data $x$ becomes a gamma distribution. Thus the $i^{\text {th }}$ case

of the posterior distribution for $\theta$ given the data $X$ can be denoted by $\mathrm{G}\left(\alpha_{i}, \beta_{i}\right)$, $i=1,2,3,4$, with pdf

$$
\pi_{i}(\theta \mid x)=\frac{\mathrm{e}^{-\beta_{i} \theta} \theta^{\alpha_{i}-1} \beta_{i}^{\alpha_{i}}}{\Gamma\left(\alpha_{i}\right)}, \quad \theta>0, \beta_{i}>0, \alpha_{i}>0
$$

for $i=1,2,3,4$.

\section{Bayes Estimator of $\theta$ and Reliability $R_{t}(\theta)$ at Time $t$}

Consider the squared error loss function defined as

$$
\mathrm{L}(\hat{\theta}, \theta)=(\hat{\theta}-\theta)^{2}
$$

The Bayes estimator of $\theta$ under the squared error loss function is nothing but the posterior mean, i.e.

$$
\hat{\theta}=\mathrm{E}_{\pi_{i}}(\theta \mid x)
$$

where $\pi_{i}$ is the posterior distribution of $\theta$ given the data $X$ in case $i$. 


\section{PATEL \& PATEL}

Under the Exponential-Gamma prior distribution, using (8) and (18), the Bayes estimators of $\theta$ and $\mathrm{R}_{t}(\theta)$ can be obtained as

$$
\begin{aligned}
\hat{\theta}_{1} & =\mathrm{E}_{\pi_{1}}(\theta \mid x) \\
& =\frac{r+a_{1}}{y+c_{1}+b_{1}}
\end{aligned}
$$

with

$$
\begin{aligned}
\mathrm{V}_{\pi_{1}}(\theta \mid x) & =\frac{\alpha_{1}}{\beta_{1}^{2}} \\
& =\frac{r+a_{1}}{\left(y+c_{1}+b_{1}\right)^{2}}
\end{aligned}
$$

and

$$
\begin{aligned}
\hat{\mathrm{R}}_{1 t}(\theta) & =\left(\frac{y+c_{1}+b_{1}}{t+y+c_{1}+b_{1}}\right)^{r+a_{1}} \\
& =\left(\frac{\beta_{1}}{t+\beta_{1}}\right)^{\alpha_{1}}
\end{aligned}
$$

with

$$
\begin{aligned}
\mathrm{V}_{\pi_{1}}\left(\mathrm{R}_{t}(\theta) \mid x\right) & =\left(\frac{\beta_{1}}{2 t+\beta_{1}}\right)^{\alpha_{1}}-\left(\frac{\beta_{1}}{t+\beta_{1}}\right)^{2 \alpha_{1}} \\
& =\left(\frac{y+c_{1}+b_{1}}{2 t+y+c_{1}+b_{1}}\right)^{r+a_{1}}-\left(\frac{y+c_{1}+b_{1}}{t+y+c_{1}+b_{1}}\right)^{2\left(r+a_{1}\right)}
\end{aligned}
$$

Similarly, the Bayes estimators of $\theta$ and $\mathrm{R}_{t}(\theta)$ at time $t$ in the case of the $i^{\text {th }}$ joint prior distribution are obtained as

$$
\hat{\theta}_{i}=\frac{\alpha_{i}}{\beta_{i}}
$$




\section{DOUBLE PRIOR SELECTION FOR EXPONENTIAL DISTRIBUTION}

with

$$
\mathrm{V}_{\pi_{i}}(\theta \mid x)=\frac{\alpha_{i}}{\beta_{i}^{2}}
$$

and

$$
\hat{\mathrm{R}}_{i t}(\theta)=\left(\frac{\beta_{i}}{t+\beta_{i}}\right)^{\alpha_{i}}
$$

with

$$
\mathrm{V}_{\pi_{i}}\left(\mathrm{R}_{t}(\theta) \mid x\right)=\left(\frac{\beta_{i}}{2 t+\beta_{i}}\right)^{\alpha_{i}}-\left(\frac{\beta_{i}}{t+\beta_{i}}\right)^{2 \alpha_{i}}
$$

for $i=2,3,4$.

\section{$(1-\alpha) 100 \%$ Equal Tail Credible Interval for $\theta$}

Let $\left[I_{1 i}, I_{2 i}\right]$ be the $(1-\alpha) 100 \%$ equal tail credible interval for $\theta$. Then $I_{1 i}$ and $I_{2 i}$ can be obtained by solving the following equations:

$$
\int_{0}^{I_{1 i}} \pi_{i}(\theta \mid x) d \theta=\frac{\alpha}{2} \text { and } \int_{I_{2 i}}^{\infty} \pi_{i}(\theta \mid x) d \theta=\frac{\alpha}{2}
$$

From (17) and (27),

$$
\mathrm{G}\left(\alpha_{i}, \beta_{i}, I_{1 i}\right)=\frac{\alpha}{2} \quad \text { and } \mathrm{G}\left(\alpha_{i}, \beta_{i}, I_{2 i}\right)=1-\frac{\alpha}{2}
$$

where

$$
\begin{aligned}
\mathrm{G}(n, a, I) & =\int_{0}^{I} \frac{\mathrm{e}^{-a \theta} \theta^{n-1} a^{n}}{\Gamma(a)} d \theta \\
& =\sum_{j=0}^{n-1} \frac{\mathrm{e}^{-I a}(I a)^{j}}{j !}
\end{aligned}
$$




\section{PATEL \& PATEL}

Thus, equation (27) reduces to

$$
\sum_{j=0}^{\alpha_{i}-1} \frac{\mathrm{e}^{-I_{1 i} \beta_{i}}\left(I_{1 i} \beta_{i}\right)^{j}}{j !}=\frac{\alpha}{2} \quad \text { and } \quad \sum_{j=0}^{\alpha_{i}-1} \frac{\mathrm{e}^{-I_{2 i} \beta_{i}}\left(I_{2 i} \beta_{i}\right)^{j}}{j !}=1-\frac{\alpha}{2}
$$

Solving these equations yields $I_{1 i}$ and $I_{2 i}, i=1,2,3,4$.

\section{Posterior Distribution of $\mathbf{R}_{t}(\boldsymbol{\theta})$}

As $\mathrm{R}_{t}(\theta)=\mathrm{e}^{-t \theta}$, from the posterior distribution of $\theta$ given the data $x$ as defined in (17), the posterior distribution of $\mathrm{R}_{t}(\theta)=\mathrm{R}$ can be derived as

$$
\pi_{i}(\mathrm{R} \mid x)=\left(\frac{\beta_{i}}{t}\right)^{\alpha_{i}} \frac{1}{\Gamma\left(\alpha_{i}\right)}(\mathrm{R})^{\frac{\beta_{i}}{t}-1}(-\ln \mathrm{R})^{\alpha_{i}-1}, \quad 0<\mathrm{R}<1
$$

The $(1-\alpha) 100 \%$ equal tail credible interval for $\mathrm{R}(t)$ can be derived by solving the equations:

$$
\int_{0}^{\mathrm{R}_{1 i}} \pi_{i}(\mathrm{R} \mid x) d \theta=\frac{\alpha}{2} \text { and } \int_{\mathrm{R}_{2 i}}^{\infty} \pi_{i}(\mathrm{R} \mid x) d \theta=\frac{\alpha}{2}
$$

From (30) and (31),

$$
\mathrm{G}\left(\alpha_{i}, \frac{\beta_{i}}{t},-\ln \mathrm{R}_{1 i}\right)=\frac{\alpha}{2} \text { and } \mathrm{G}\left(\alpha_{i}, \frac{\beta_{i}}{t},-\ln \mathrm{R}_{2 i}\right)=1-\frac{\alpha}{2}
$$

Again using (28), deduce that

$$
\sum_{j=0}^{\alpha_{i}-1} \frac{\left(\mathrm{R}_{1 i}\right)^{\frac{\beta_{i}}{t}}\left(-\ln \mathrm{R}_{1 i} \frac{\beta_{i}}{t}\right)^{j}}{j !}=\frac{\alpha}{2} \text { and } \sum_{j=0}^{\alpha_{i}-1} \frac{\left(\mathrm{R}_{2 i}\right)^{\frac{\beta_{i}}{t}\left(-\ln \mathrm{R}_{2 i} \frac{\beta_{i}}{t}\right)^{j}}}{j !}=1-\frac{\alpha}{2}
$$

Finally, solving these equations, obtain $\mathrm{R}_{1 i}$ and $\mathrm{R}_{2 i}$ for $i=1,2,3,4$. 


\section{Bayes Predictive Estimator and Equal Tail Credible Interval for a Future Observation}

A predictive estimator is derived for a future observation and its equal tail credible interval. Let $Z_{i}$ be a future observation which has already survived $X_{(r)}$, and let $W_{(i)}=Z_{i}-X_{(r)}$. Given the data $x$, the conditional joint pdf of $W_{i}$ and $\theta$ is

$$
\mathrm{h}_{i}\left(w_{i}, \theta \mid x\right)=\mathrm{f}\left(w_{i}, \theta \mid x\right) \pi_{i}(\theta \mid x)
$$

From (10) and (17),

$$
\mathrm{h}_{i}\left(w_{i}, \theta \mid x\right)=\frac{\beta_{i}^{\alpha_{i}}}{\Gamma\left(\alpha_{i}\right)} \mathrm{e}^{-\theta\left(w_{i}+\beta_{i}\right)} \theta^{\alpha_{i}}
$$

Integrating out with respect to $\theta$, the predictive density of $w_{i}$ under the $i^{\text {th }}$ case of the joint prior distribution comes out as

$$
\begin{aligned}
\mathrm{p}_{i}\left(w_{i} \mid x\right) & =\int_{0}^{\infty} \frac{\beta_{i}^{\alpha_{i}}}{\Gamma\left(\alpha_{i}\right)} \mathrm{e}^{-\theta\left(w_{i}+\beta_{i}\right)} \theta^{\alpha_{i}} d \theta \\
& =\frac{\alpha_{i} \beta_{i}^{\alpha_{i}}}{\left(w_{i}+\beta_{i}\right)^{\alpha_{i}+1}}, \quad w_{i}>0, \alpha_{i}>0, \beta_{i}>0, i=1,2,3,4
\end{aligned}
$$

The Bayes estimator of $w_{i}$ under a squared error loss function is given by

$$
\begin{aligned}
w_{i}^{*} & =\mathrm{E}\left(w_{i} \mid x\right)=\alpha_{i} \beta_{i}^{\alpha_{i}} \int_{0}^{\infty} \frac{w_{i}}{\left(w_{i}+\beta_{i}\right)^{\alpha_{i}+1}} d w_{i} \\
& =\frac{\beta_{i}}{\alpha_{i}-1}, \quad i=1,2,3,4
\end{aligned}
$$

Hence

$$
z_{i}^{*}=x_{(r)}+w_{i}^{*}
$$

Now the $(1-\alpha) 100 \%$ predictive interval $\left(h_{1 i}, h_{2 i}\right)$ for $w_{i}$ can be obtained by solving the equations 


$$
\int_{0}^{h_{1 i}} \mathrm{p}_{i}\left(w_{i} \mid x\right) d w_{i}=\frac{\alpha}{2} \quad \text { and } \quad \int_{0}^{h_{2 i}} \mathrm{p}_{i}\left(w_{i} \mid x\right) d w_{i}=1-\frac{\alpha}{2}
$$

Using (34) in (37), after some algebraic manipulation,

$$
h_{1 i}=\beta_{i} \frac{1-\left(1-\frac{\alpha}{2}\right)^{\frac{1}{\alpha_{i}}}}{\left(\frac{\alpha}{2}\right)^{\frac{1}{\alpha_{i}}}} \text { and } \quad h_{2 i}=\beta_{i} \frac{1-\left(\frac{\alpha}{2}\right)^{\frac{1}{\alpha_{i}}}}{\left(\frac{\alpha}{2}\right)^{\frac{1}{\alpha_{i}}}}, \quad i=1,2,3,4
$$

\section{Prediction of the Remaining $(n-r)$ Failure Times Truncated at $X_{(r)}$ and their Equal Tail Intervals}

Consider the prediction of the remaining $(n-r)$ failure times given the first $r$ failure times of a sample of $n$ units. Let $x_{(s) i},(r+1 \leq s \leq n)$ denote the failure times of the $s^{\text {th }}$ unit to fail under the $i^{\text {th }}$ case of double prior distribution. The conditional pdf of $u=x_{(s)}-x_{(r)}$ from a pdf truncated at $x_{(r)}$ is given by

$$
\mathrm{f}(u \mid \theta)=\frac{(\mathrm{f}(u))^{s-r-1}[1-\mathrm{F}(u)]^{n-s} \mathrm{f}(u)}{\beta_{(s-r, n-s+1)}}, \quad u \geq 0
$$

Here $\mathrm{f}(u)$ and $\mathrm{F}(u)$ are the pdf and cdf of a random variable $X$, respectively, as given in (1) and (2). Hence

$$
\mathrm{f}(u \mid \theta)=\frac{\left(1-\mathrm{e}^{-\theta u}\right)^{s-r-1}\left[\mathrm{e}^{-\theta u}\right]^{n-s} \theta \mathrm{e}^{-\theta u}}{\beta_{(s-r, n-s+1)}}, \quad u \geq 0, r+1 \leq s \leq n
$$

Given $x$, the conditional joint pdf of $u$ and $\theta$ under the $i^{\text {th }}$ case of the double prior distribution is given by 


$$
\begin{aligned}
\mathrm{f}_{i}\left(u_{i}, \theta \mid x\right) & =\mathrm{f}\left(u_{i} \mid \theta\right) \pi_{i}(\theta \mid x) \\
& =\frac{\left(1-\mathrm{e}^{-\theta u_{i}}\right)^{s-r-1}\left[\mathrm{e}^{-\theta u_{i}}\right]^{n-s} \theta \mathrm{e}^{-\theta u_{i}}}{\beta_{(s-r, n-s+1)}} \frac{\beta_{i}^{\alpha_{i}}}{\Gamma\left(\alpha_{i}\right)} \mathrm{e}^{-\theta \beta_{i}} \theta^{\alpha_{i}-1} \\
& =\frac{\beta_{i}^{\alpha_{i}}}{\Gamma\left(\alpha_{i}\right) \beta_{(s-r, n-s+1)}} \sum_{j=0}^{s-r-1} \frac{(-1)^{j}(s-r-1) !}{j !(s-r-j-1) !} \mathrm{e}^{-\theta\left(j u_{i}+u_{i}(n-s+1)+\beta_{i}\right)} \theta^{\alpha_{i}}
\end{aligned}
$$

Integrating out $\theta$, the predictive density of $u_{i}$ for the $i^{\text {th }}$ case of the double prior is given by

$$
\begin{aligned}
& \mathrm{p}_{i}\left(u_{i} \mid x\right)= \\
& \quad \frac{\beta_{i}^{\alpha_{i}}}{\Gamma\left(\alpha_{i}\right) \beta_{(s-r, n-s+1)}} \sum_{j=0}^{s-r-1} \frac{(-1)^{j}(s-r-1) !}{j !(s-r-j-1) !} \frac{\Gamma\left(\alpha_{i}+1\right)}{\left[j u_{i}+u_{i}(n-s+1)+\beta_{i}\right]^{\alpha_{i}+1}}
\end{aligned}
$$

with $u_{i}>0$. Under the squared error loss function, the Bayes predictive estimator of $u_{i}$ is

$$
\begin{aligned}
u_{i}^{*} & =\mathrm{E}\left(u_{i} \mid x\right) \\
& =\int_{0}^{\infty} u_{i} \mathrm{p}_{i}\left(u_{i} \mid x\right) d u_{i}
\end{aligned}
$$

On simplification,

$$
u_{i}^{*}=\frac{\alpha_{i} \beta_{i}}{\beta_{(s-r, n-s+1)}} \sum_{j=0}^{s-r-1} \frac{(-1)^{j}(s-r-1) ! \beta_{\left(2, \alpha_{i}-1\right)}}{j !(s-r-j-1) !(j+n-s+1)^{2}}, \quad i=1,2,3,4
$$

For $s=r+1$, obtain the estimator for the $x_{(r+1)}^{\text {th }}$ failure time as $x_{(r+1)}=u_{i}^{*}+x_{(r)}$, where

$$
u_{i}^{*}=\frac{\beta_{i}}{\left(\alpha_{i}-1\right)(n-r)}, \quad i=1,2,3,4
$$

Similarly, for $s=r+2, r+3, \ldots, n, u_{i}^{*}$ can be obtained. Now a $(1-\alpha) 100 \%$ equal tail confidence interval $\left(H_{1 i}, H_{2 i}\right)$ for $u_{i}$ is the solution of the equations: 


$$
\int_{0}^{H_{1 i}} \mathrm{p}_{i}\left(u_{i} \mid x\right) d u_{i}=\frac{\alpha}{2} \quad \text { and } \quad \int_{0}^{H_{2 i}} \mathrm{p}_{i}\left(u_{i} \mid x\right) d u_{i}=1-\frac{\alpha}{2}
$$

Using (41) and (44), we have

$$
\frac{1}{\beta_{(s-r, n-s+1)}} \sum_{j=0}^{s-r-1} \frac{(-1)^{j}(s-r-1) !}{j !(s-r-j-1) !}\left[1-\frac{\beta_{i}^{\alpha_{i}}}{\left\{H_{1 i}(j+n-s+1)+\beta_{i}\right\}^{\alpha_{i}}}\right]=\frac{\alpha}{2}
$$

and

$$
\frac{1}{\beta_{(s-r, n-s+1)}} \sum_{j=0}^{s-r-1} \frac{(-1)^{j}(s-r-1) !}{j !(s-r-j-1) !}\left[1-\frac{\beta_{i}^{\alpha_{i}}}{\left\{H_{2 i}(j+n-s+1)+\beta_{i}\right\}^{\alpha_{i}}}\right]=1-\frac{\alpha}{2}
$$

Solving the equations in $(45)$, obtain the $(1-\alpha) 100 \%$ equal tail confidence interval $\left(H_{1 i}, H_{2 i}\right)$ for the remaining $(n-r)$ failure times given the first $r$ failure times of a sample of size $n$ for the $i^{\text {th }}$ case of the double prior distribution, $i=1,2$, 3,4 .

\section{A Real Data Example}

The data were obtained from Bain and Engelhardt (1991), representing the times between successive failures. The times are exponentially distributed (Kolmogorov-Smirnov $p$-value: 0.900) with mean failure time 3.744.

Times between system failures data:

$5.2,8.4,0.9,0.1,5.9,17.9,3.6,2.5,1.2,1.8,1.8,6.1,5.3,1.2,1.2,3.0,3.5$, $7.6,3.4,0.5,2.4,5.3,1.9,2.8,0.1$

For this example, Bayes estimates of parameter $\theta$, reliability $\mathrm{R}(t)$, Bayes predictive estimator of a future observation $Z^{*}$, and predictive estimator of the remaining order statistic $x_{(r+1)}$ based on the known first $r$ order statistics and their equal tail credible intervals ( in the bracket) are derived under different types of joint prior distributions and presented in Table 1 for hyper parametric values $a_{i}=b_{i}=c_{i}=4, i=1,2,3,4$. 


\section{DOUBLE PRIOR SELECTION FOR EXPONENTIAL DISTRIBUTION}

Table 1. Bayes estimates and credible intervals

\begin{tabular}{rrrrr} 
Prior Distribution & $\hat{\boldsymbol{\theta}}$ & $\hat{\mathbf{R}}(\boldsymbol{t})$ & $\boldsymbol{Z}$ & $\boldsymbol{X}(r+1)$ \\
\hline Exponential-Gamma & 0.291971 & 0.242397 & 8.873913 & 6.014783 \\
& $(0.187679$, & $(0.122587$, & $(5.386759$, & $(5.303434$, \\
& $0.419851)$ & $0.92453)$ & $18.95711)$ & $9.361143)$ \\
Gamma-Chi-square & 0.317662 & 0.214404 & 8.579167 & 5.955853 \\
& $(0.205576$, & $(0.103450$, & $(5.379741$, & $(5.303156$, \\
& $0.453757)$ & $0.357776)$ & $17.81308)$ & $9.015658)$ \\
Chi-square-Exponential & 0.279543 & 0.257919 & 9.047619 & 6.049524 \\
& $(0.175190$, & $(0.130106$, & $(5.390621$, & $(5.303587$, \\
& $0.407894)$ & $0.416476)$ & $19.66698)$ & $9.586129)$ \\
Only Gamma & 0.306905 & 0.225945 & 8.700000 & 5.980000 \\
& $(0.196647$, & $(0.110079$, & $(5.382537$, & $(5.303267$, \\
& $0.441330)$ & $0.374119)$ & $18.29253)$ & $9.16352)$ \\
\hline
\end{tabular}

\section{Simulation Study}

A Monte Carlo simulation study was carried out to compare the performance of the Bayes estimators under different joint priors and single prior. To generate 1000 Type II censored samples, the value of the parameter $\theta$ is considered as 0.7 and the values of the hyper parameters for all joint and single priors are considered to be $a_{i}=b_{i}=c_{i}=4$. The reliability is calculated at time $t=1$.

Values of the hyper parameters can be obtained from our prior belief. If there is any information from past data about the mean, variance, or about reliability measure, by comparing such prior beliefs with the theoretical results and by solving the equations the estimates of the hyper parameters can be obtained. A value 4 was used for the hyper parameters, i.e. $a_{i}=b_{i}=c_{i}=4$, for simulation purpose.

The simulation was conducted for different values of sample size $(n)$ and of fixed censored value $(r):(n, r)=(15,5),(15,10),(15,12),(30,10),(30,20)$, and $(30,24)$. In each case, Bayes estimates of $\theta, \mathrm{R}(t)$, future observation $z^{*}$, and the $(r+1)^{\text {th }}$ ordered failure time $X_{(r+1)}$ are derived. Their mean square errors (MSE) and Bayes equal tail credible intervals are also obtained. The first, second, and third values for each cell of the third and fourth columns of Tables 2 to 5 denote the Bayes estimate, MSE, and credible intervals, respectively. 
PATEL \& PATEL

Table 2. Bayes estimates and credible intervals for $\theta$ and $R(t)$ for $n=15$

\begin{tabular}{|c|c|c|c|}
\hline Joint priors & $r$ & $\theta$ & $\mathbf{R}(t)$ \\
\hline \multirow[t]{9}{*}{ Exponential-Gamma } & 5 & 0.620546 & 0.552537 \\
\hline & & 0.020495 & 0.006874 \\
\hline & & $(0.283758,1.08687)$ & $(0.344604,0.754072)$ \\
\hline & 10 & 0.658255 & 0.529796 \\
\hline & & 0.018407 & 0.005175 \\
\hline & & $(0.359880,1.045241)$ & $(0.358859,0.699491)$ \\
\hline & 12 & 0.666924 & 0.524325 \\
\hline & & 0.017280 & 0.004657 \\
\hline & & $0.381210,1.031245)$ & $(0.363315,0.684830)$ \\
\hline \multirow[t]{9}{*}{ Gamma-Chi-square } & 5 & 0.923133 & 0.423851 \\
\hline & & 0.105171 & 0.012966 \\
\hline & & $(0.442683,1.577159)$ & $(0.223643,0.646348)$ \\
\hline & 10 & 0.852047 & 0.444890 \\
\hline & & 0.064292 & 0.009208 \\
\hline & & $0.476877,1.334254)$ & $(0.276191,0.624641)$ \\
\hline & 12 & 0.835802 & 0.449584 \\
\hline & & 0.054266 & 0.008109 \\
\hline & & $(0.486891,1.277455)$ & $(0.289965,0.618192)$ \\
\hline \multirow[t]{9}{*}{ Chi-square-Exponential } & 5 & 0.646193 & 0.545833 \\
\hline & & 0.030033 & 0.008780 \\
\hline & & $(0.259808,1.205569)$ & $(0.313306,0.772884)$ \\
\hline & 10 & 0.681619 & 0.521700 \\
\hline & & 0.026693 & 0.006481 \\
\hline & & $(0.352208,1.117977)$ & $(0.338067,0.705574)$ \\
\hline & 12 & 0.688308 & 0.516572 \\
\hline & & 0.024432 & 0.005762 \\
\hline & & $(0.376310,1.092958)$ & $(0.345085,0.688844)$ \\
\hline \multirow[t]{9}{*}{ Only Gamma } & 5 & 0.873913 & 0.444968 \\
\hline & & 0.085159 & 0.010865 \\
\hline & & $(0.399614,1.530634)$ & $(0.234776,0.674373)$ \\
\hline & 10 & 0.819872 & 0.459206 \\
\hline & & 0.055039 & 0.008196 \\
\hline & & $(0.448236,1.301867)$ & $(0.285404,0.642552)$ \\
\hline & 12 & 0.807536 & 0.462281 \\
\hline & & 0.046925 & 0.007281 \\
\hline & & $(0.461582,1.248669)$ & $(0.298505,0.633848)$ \\
\hline
\end{tabular}




\section{DOUBLE PRIOR SELECTION FOR EXPONENTIAL DISTRIBUTION}

Table 3. Bayes estimates and credible intervals for $\theta$ and $\mathrm{R}(t)$ for $n=30$

\begin{tabular}{|c|c|c|c|}
\hline Joint priors & $r$ & $\boldsymbol{\theta}$ & $\mathrm{R}(t)$ \\
\hline \multirow[t]{8}{*}{ Exponential-Gamma } & 10 & 0.658399 & 0.529698 \\
\hline & & $\begin{array}{r}0.018254 \\
(0.359958,1.045468)\end{array}$ & $\begin{array}{r}0.005154 \\
(0.358739,0.699423)\end{array}$ \\
\hline & 20 & 0.681753 & 0.513881 \\
\hline & & 0.013730 & 0.003502 \\
\hline & & $(0.436817,0.980345)$ & $(0.380295,0.647858)$ \\
\hline & 24 & 0.686436 & 0.510669 \\
\hline & & 0.012823 & $\begin{array}{r}0.003193 \\
0.535486\end{array}$ \\
\hline & & $(0.456137,0.963065)$ & $(0.386387,0.635486)$ \\
\hline \multirow[t]{9}{*}{ Gamma-Chi-square } & 10 & 0.852159 & 0.444759 \\
\hline & & 0.063765 & 0.009202 \\
\hline & & $(0.476952,1.334466)$ & $(0.275997,0.624550)$ \\
\hline & 20 & 0.791372 & 0.463756 \\
\hline & & 0.030889 & 0.005325 \\
\hline & & $(0.512140,1.130405)$ & $(0.330128,0.602004)$ \\
\hline & 24 & 0.779839 & 0.467613 \\
\hline & & 0.026108 & 0.004659 \\
\hline & & $(0.522275,1.088224)$ & $(0.343117,0.595763)$ \\
\hline \multirow[t]{9}{*}{ Chi-square-Exponential } & 10 & 0.681728 & 0.521585 \\
\hline & & 0.026326 & 0.006450 \\
\hline & & $(0.352264,1.118155)$ & $(0.337909,0.705506)$ \\
\hline & 20 & 0.696408 & 0.508011 \\
\hline & & 0.017468 & 0.004104 \\
\hline & & $(0.436440,1.016152)$ & $(0.368543,0.648527)$ \\
\hline & 24 & 0.699166 & 0.505450 \\
\hline & & 0.015863 & 0.003687 \\
\hline & & $(0.456723,0.992416)$ & $(0.376460,0.635478)$ \\
\hline \multirow[t]{9}{*}{ Only Gamma } & 10 & 0.819986 & 0.459075 \\
\hline & & 0.054456 & 0.008184 \\
\hline & & $(0.448299,1.302049)$ & $(0.285213,0.642467)$ \\
\hline & 20 & 0.772391 & 0.472523 \\
\hline & & 0.027461 & 0.004911 \\
\hline & & $(0.494890,1.110679)$ & $(0.336687,0.612383)$ \\
\hline & 24 & 0.763553 & 0.475196 \\
\hline & & 0.023514 & 0.004341 \\
\hline & & $(0.507380,1.071258)$ & $(0.348968,0.604628)$ \\
\hline
\end{tabular}




\section{PATEL \& PATEL}

Table 4. Bayes estimates and credible intervals for $Z Z$ and $X(r+1)$ for $n=15$

\begin{tabular}{|c|c|c|c|}
\hline Joint priors & $r$ & $Z \mathbf{Z}$ & $X(r+1)$ \\
\hline \multirow[t]{9}{*}{ Exponential-Gamma } & 5 & 2.435166 & 0.738994 \\
\hline & & 0.394374 & 0.078934 \\
\hline & & $(0.593002,8.189055)$ & $(0.550949,1.976656)$ \\
\hline & 10 & 3.161177 & 1.800628 \\
\hline & & 0.670290 & 0.303256 \\
\hline & & $(1.50051,8.125572)$ & $(1.462075,3.494605)$ \\
\hline & 12 & 3.738878 & 2.633385 \\
\hline & & 0.880619 & 0.541882 \\
\hline & & $(2.120031,8.530325)$ & $(2.084978,4.972641)$ \\
\hline \multirow[t]{9}{*}{ Gamma-Chi-square } & 5 & 1.836860 & 0.6791650 .076552 \\
\hline & & 0.342344 & 0.076552 \\
\hline & & $(0.579878,5.715377)$ & $(0.550820,1.500505)$ \\
\hline & 10 & 2.789699 & 1.726333 \\
\hline & & 0.631423 & 0.298065 \\
\hline & & $(1.491927,6.648723)$ & $(1.461736,3.037210)$ \\
\hline & 12 & 3.416487 & 2.525922 \\
\hline & & 0.845020 & 0.532755 \\
\hline & & $(2.112493,7.260214)$ & $(2.084148,4.398042)$ \\
\hline \multirow[t]{9}{*}{ Chi-square-Exponential } & 5 & 2.480049 & 0.743482 \\
\hline & & 0.572588 & 0.086304 \\
\hline & & $(0.592479,83.582894)$ & $(0.5506944,2.117547)$ \\
\hline & 10 & 3.152210 & 1.798834 \\
\hline & & 0.774620 & 0.316687 \\
\hline & & $(1.499793,8.157714)$ & $(1.462047,3.526374)$ \\
\hline & 12 & 3.724764 & 2.628682 \\
\hline & & 0.971637 & 0.564728 \\
\hline & & $(2.119327,8.524054)$ & $(2.084899,4.985378)$ \\
\hline \multirow[t]{9}{*}{ Only Gamma } & 5 & 1.935169 & 0.688994 \\
\hline & & 0.394375 & 0.078935 \\
\hline & & $(0.581735,6.162530)$ & $(0.55084,1.598301)$ \\
\hline & 10 & 2.853482 & 1.739090 \\
\hline & & 0.670290 & 0.303256 \\
\hline & & $(1.493269,6.919713)$ & $(1.461789,3.1265887)$ \\
\hline & 12 & 3.472212 & 2.544495 \\
\hline & & 0.880620 & 0.548882 \\
\hline & & $(2.113695,7.493128)$ & $(2.084279,4.507569)$ \\
\hline
\end{tabular}




\section{DOUBLE PRIOR SELECTION FOR EXPONENTIAL DISTRIBUTION}

Table 5. Bayes estimates and credible intervals for $Z Z$ and $X_{(r+1)}$ for $n=30$

\begin{tabular}{|c|c|c|c|}
\hline Joint priors & $r$ & $Z ゙$ & $\boldsymbol{X}(r+1)$ \\
\hline \multirow[t]{9}{*}{ Exponential-Gamma } & 10 & 2.259726 & 0.644702 \\
\hline & & 0.268444 & 0.037871 \\
\hline & & $(0.599704,7.222201)$ & $(0.559800,1.235965)$ \\
\hline & 20 & 3.081523 & 1.663868 \\
\hline & & 0.377836 & 0.142476 \\
\hline & & $(1.544589,7.525605)$ & $(1.506728,2.533688)$ \\
\hline & 24 & 3.735099 & 2.442472 \\
\hline & & 0.521211 & 0.273523 \\
\hline & & $(2.221829,8.081593)$ & $(2.184982,3.693112)$ \\
\hline \multirow[t]{9}{*}{ Gamma-Chi-square } & 10 & 1.888297 & 0.626132 \\
\hline & & 0.243800 & 0.037402 \\
\hline & & $(0.591122,5.745548)$ & $(0.559779,1.081897)$ \\
\hline & 20 & 2.870057 & 1.642720 \\
\hline & & 0.364330 & 0.141661 \\
\hline & & $(1.53951,6.710169)$ & $(1.506678,2.392701)$ \\
\hline & 24 & 3.554705 & 2.412403 \\
\hline & & 0.508779 & 0.272072 \\
\hline & & $(2.217467,7.390260)$ & $(2.184864,3.514660)$ \\
\hline \multirow[t]{9}{*}{ Chi-square-Exponential } & 10 & 2.250640 & 0.644248 \\
\hline & & 0.336536 & 0.039082 \\
\hline & & $(0.598986,7.253850)$ & $(0.559799,1.253045)$ \\
\hline & 20 & 3.064872 & 1.662202 \\
\hline & & 0.409659 & 0.144348 \\
\hline & & $(1.544036,7.481150)$ & $(1.506722,2.530874)$ \\
\hline & 24 & 3.719194 & 2.439816 \\
\hline & & 0.549649 & 0.276788 \\
\hline & & $(2.221333,8.034713)$ & $(2.184969,3.685021)$ \\
\hline \multirow[t]{9}{*}{ Only Gamma } & 10 & 1.952034 & 0.629318 \\
\hline & & 0.268442 & 0.037872 \\
\hline & & $(0.592464,6.016336)$ & $(0.559782,1.113566)$ \\
\hline & 20 & 2.907609 & 1.646474 \\
\hline & & 0.377836 & 0.142476 \\
\hline & & $(1.540366,6.861025)$ & $(1.506685,2.420264)$ \\
\hline & 24 & 3.586950 & 2.417776 \\
\hline & & 0.521211 & 0.273523 \\
\hline & & $(2.218210,7.518306)$ & $(2.184883,3.548975)$ \\
\hline
\end{tabular}


PATEL \& PATEL

\section{Conclusion}

\section{Comparison of Priors Based on the MSE and Credible Interval of $\boldsymbol{\theta}$}

From the third columns of Tables 2 and 3 it is observed that the values of the MSE of the Bayes estimator of parameter $\theta$ and length of its credible intervals are smaller in the case of the Exponential-Gamma joint prior and then followed by Chi-square-Exponential, Gamma, and Gamma-Chi-square priors in all the values of $n$ and $r$ considered here.

\section{Comparison of Priors Based on the MSE and Credible Interval of $\mathbf{R}(t)$}

From the fourth column of Tables 2 and 3 it is observed that, for all values of $n$ and $r$ considered here, the values of the MSE of the Bayes estimator of $\mathrm{R}(t)$ are smaller in the case of the joint prior Exponential-Gamma, and then followed by Chi-square-Exponential, Gamma, and Gamma-Chi-square priors. The Exponential-Gamma joint prior generates the minimum length of the credible intervals of $\mathrm{R}(t)$, followed by Gamma-Chi-square, Gamma, and Chi-squareExponential priors in all the values of $n$ and $r$ considered here.

\section{Comparison of Priors Based on MSE and Credible Interval of Future Predicted Value}

From the third columns of Tables 4 and 5, note that MSEs and lengths of the credible intervals are minimum in the case of the Gamma-Chi-square prior, and then followed by Gamma, Exponential-Gamma, and Chi-square-Exponential priors in all the values of $n$ and $r$ considered here.

\section{Comparison Based on the MSE and Credible Interval of Next Ordered Failure Time $X_{(r+1)}$}

From the fourth columns of the Tables 4 and 5, we observed that the minimum MSE as well as the minimum length of credible interval are generated by the Gamma-Chi-square joint prior, whereas other priors give erratic effect.

Thus, it was found that the Exponential-Gamma joint prior performs well compared to the other single and joint priors considered in this study. 


\section{Acknowledgements}

The authors are thankful to the honorable referees and to the editor of the journal for making good comments on the paper.

\section{References}

Bain, L. J., \& Engelhardt, M. (1991). Statistical analysis of reliability and life testing models. New York, NY: Marcel Dekker.

Balakrishnan, N., \& Cohen, A. C. (1991). Order statistics and inference: Estimation methods. Boston, MA: Academic Press.

Bartholomew, D. J. (1957). A problem in life testing. Journal of the American Statistical Association, 52(279), 350-355. doi: 10.2307/2280905

Box, G. E. P., \& Tiao, G. C. (1973). Bayesian inference in statistical analysis. Reading, MA: Addition-Wesley.

Dunsmore, I. R. (1974). The Baysian predictive distribution in life testing. Technometrics, 16(3), 455-466. doi: 10.1080/00401706.1974.10489216

Epstein, B. (1954). Truncated life tests in the exponential case. The Annals of Mathematical Statistics, 25(3), 555-564. doi: 10.1214/aoms/1177728723

Epstein, B. (1960a). Tests for the validity of the assumption that the underlying distribution of life is exponential. Part I. Technometrics, 2(1), 83-101. doi: $10.2307 / 1266533$

Epstein, B. (1960b). Tests for the validity of the assumption that the underlying distribution of life is exponential. Part II. Technometrics, 2(1), 167183. doi: $10.2307 / 1266543$

Epstein, B., \& Sobel, M. (1953). Life testing. Journal of the American Statistical Association, 48(263), 486-502. doi: 10.2307/2281004

Epstein, B., \& Sobel, M. (1954). Some problems relevant to life testing from an exponential distribution. The Annals of Mathematical Statistics, 25(2), 373-381. doi: 10.1214/aoms/1177728793

Evans, I. G., \& Nigm, A. H. M. (1980). Bayesian prediction for the left truncated exponential distribution. Technometrics, 22(2), 201-204. doi: $10.2307 / 1268459$

Hahn, G. J. (1975). A Simultaneous prediction limit on the mean of future samples from an exponential distribution. Technometrics, 17(3), 341-345. doi: $10.2307 / 1268071$ 


\section{PATEL \& PATEL}

Haq, A., \& Aslam, M. (2009). On the double prior selection for the parameter of the parameter of Poisson distribution. InterStat: Statistics on the Internet, 2009(November), \#1. Retrieved from http://interstat.statjournals.net/YEAR/2009/articles/0911001.pdf

Haq, A., \& Dey, S. (2011). Bayesian estimation of Erlang distribution under different prior distributions. Journal of Reliability and Statistical Studies, 4(1), 130 .

Howlader, H. A., \& Hossain, A. (1995). On Bayesian estimation and prediction from Rayleigh based on Type II censored data. Communications in Statistics - Theory and Methods, 24(9), 2249-2259. doi:

10.1080/03610929508831614

Lawless, J. F. (1971). A prediction problem concerning samples from the exponential distribution, with applications in life testing. Technometrics, 13(4), 725-730. doi: 10.2307/1266949

Likeš, J. (1974). Prediction of sth ordered observations for the twoparameter exponential distribution. Technometrics, 16(2), 241-244. doi: $10.2307 / 1267945$

Mann, N. R., Schafer, R. E., \& Singpurwalla, N. D. (1974). Methods for statistical analysis of reliability and life data. New York, NY: John Wiley and Sons.

Radha, R. K., \& Vekatesan, P. V. (2013). On the double prior selection for the parameter of Maxwell distribution. International Journal of Scientific \& Engineering Research, 4(5), 1238-1241. Retrieved from http://www.ijser.org/researchpaper\%5CON-THE-DOUBLE-PRIOR-

SELECTION-FOR-THE-PARAMETER-OF-MAXWELL-DISTRIBUTION.pdf

Saleem, M., \& Aslam, M. (2008). On the prior selection for the mixture of Rayleigh distribution using predictive intervals. Pakistan Journal of Statistics, 24(1), 21-35.

Tahir, M., \& Zawar, H. (2008). Comparison of non-informative priors for number of defect (Poisson) model. InterStat: Statistics on the Internet, 2008(April), \#2. Retrieved from http://interstat.statjournals.net/YEAR/2008/articles/0804002.pdf 